\title{
Odontogenic Myxoma -Report Of A Rare Case
}

\author{
Sangeetha $\mathrm{K} \mathrm{P}^{1}$, Tinky Bose $\mathrm{C}^{2}$, Anita Balan ${ }^{3}$, Sandhya $\mathrm{K} \mathrm{N}^{4}$ \\ ${ }^{I}$ (P.G student, Oral Medicine and Radiology, Government Dental College, Trivandrum/ KUHS, India) \\ ${ }_{2}^{2}$ (Professor \& Head, Oral Medicine and Radiology, Government Dental College, Trivandrum/ KUHS, India) \\ ${ }_{3}^{3}$ (Principal, Government Dental College, Alappuzha/ KUHS, India) \\ ${ }^{4}$ (P.G student, Oral Medicine and Radiology, Government Dental College, Trivandrum/ KUHS, India)
}

\begin{abstract}
Odontogenic myxoma is an uncommon, benign, locally invasive neoplasm arising from embryonic mesenchyme of dental primordium and exclusively present in tooth bearing areas of the jaws. It is most often seen in females of 20-40 years of age and mainly affects the posterior mandible. The lesion remains asymptomatic until it reaches a noticeable size. The radiographic features are variable. This article presents a case of odontogenic myxoma presenting in an unusual site and age with classic radiographic appearance. The clinical, radiological, histopathological features and surgical dilemmas in managing the same is discussed.
\end{abstract}

Keywords: Odontogenic myxoma, Myxofibroma, Myxoma, Odontogenic tumour, Ectomesenchyme

\section{Introduction}

Odontogenic myxoma is a slowly growing, asymptomatic, locally invasive, benign tumour which accounts for only 3-6\% of total odontogenic tumours.[1-3] It is most commonly seen in females of second to fourth decades of life, frequently in posterior mandibular region. Even though it exhibits bizarre radiographic appearances, the typical internal trabeculations intersecting at right angles forming straight, fine, elongated and lacy loculations inspired Eversole to term it as 'Lichen planus of jaw bone'.[4] WHO considers it as a benign tumour of ectomesenchymal origin with or without odontogenic epithelium.[3]Treatment is considered to be wide surgical excision and long term follow-up owing to its aggressive nature and higher incidence of reccurence.[5]

\section{Case Report}

A 61 year old male patient presented with an asymptomatic swelling in lower front gums of 6 months. He gave a history of exfoliation of lower front teeth 6 months back following which he noticed a swelling which gradually increased in size. There was no history of pain or pareasthesia. He was a known diabetic, hypertensive and dyslipidemic and was under treatment. On extraoral examination there was no visible or palpable swelling. (Fig 1). On intraoral examination, a well defined swelling of size approximately $2 \times 2 \mathrm{~cm}$ was noted in the edentulous alveolar ridge of 32, 31, 41 and 42 region obliterating the labial vestibule and floor of mouth. (Fig 2).Mucosa overlying the lesion was smooth, intact and was having almost same colour as that of adjacent mucosa. Oral hygiene was poor with deposits of calculus noted over the adjacent teeth. On palpation, the swelling was non tender, firm in consistency in superior region where as soft in the inferior region both labially and lingually. 33 and 43 were Grade III mobile. A provisional diagnosis of benign odontogenic tumour or Odontogenic cyst probably residual cyst was made. Aspiration was done to rule out possibility of cyst. It was negative.

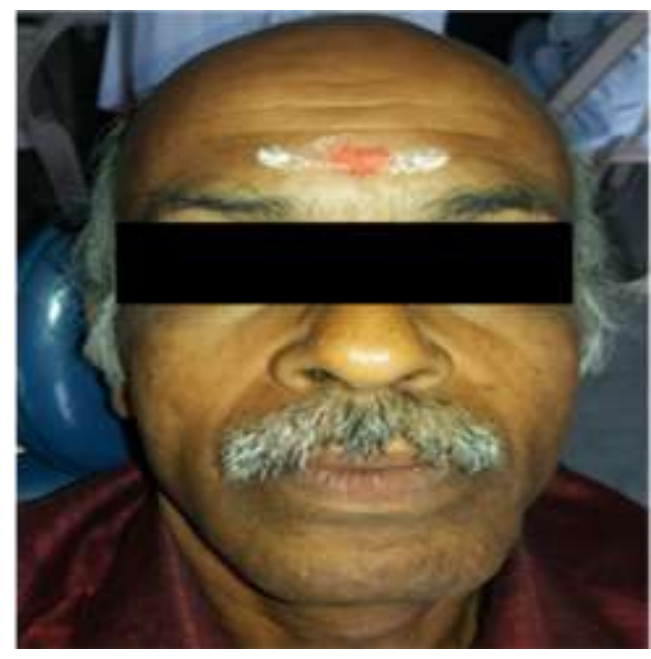


Fig.1 Extraoral photograph showing no visible swelling

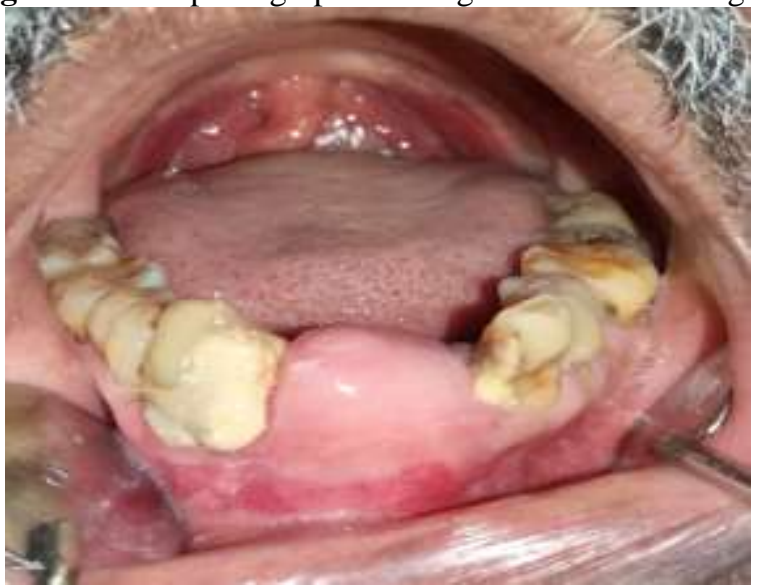

Fig.2 Intraoral photograph showing well defined swelling in alveolar ridge of 32-42 region.

Panoramic radiograph showed a multilocular radiolucency of size approximately $5 \times 4 \mathrm{~cm}$ in mandibular symphyseal parasymphyseal region, crossing the midline, extending from 35 to 44 region with well defined corticated border inferiorly. The lesion was approximately $0.5 \mathrm{~cm}$ above the lower border of the mandible and superiorly it perforated the superior border of mandibular alveolar ridge. There was no root resorption or displacement of adjacent tooth (Fig 3).

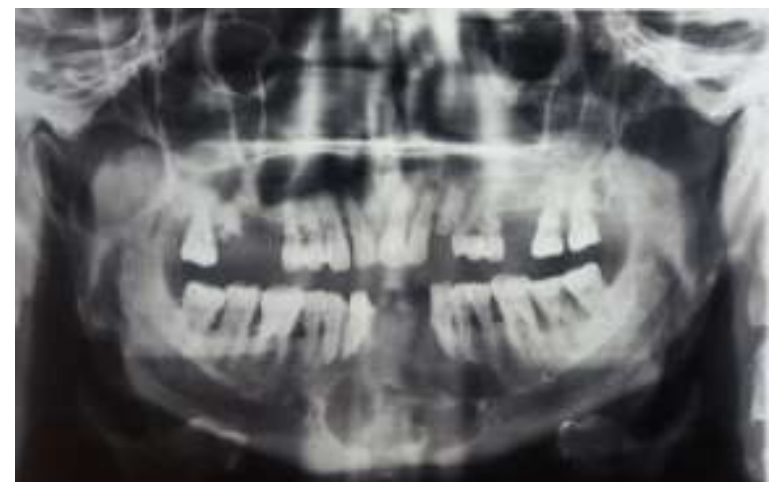

Fig 3: Panoramic radiograph showing multilocular radiolucency in 35-44 region

Anterior mandibular topographic occlusal view showed well defined straight, thin and sharp angular internal loculations which were more pronounced towards the inferior border resembling a tennis racket pattern. Superior border was perforated. (Fig 4) No obvious labial or lingual cortical plate expansion was noted in anterior mandibular true occlusal radiograph. (Fig 5). Based upon the radiographic findings, a differential diagnosis of odontogenic myxoma, ameloblastoma and central giant cell granuloma were made.

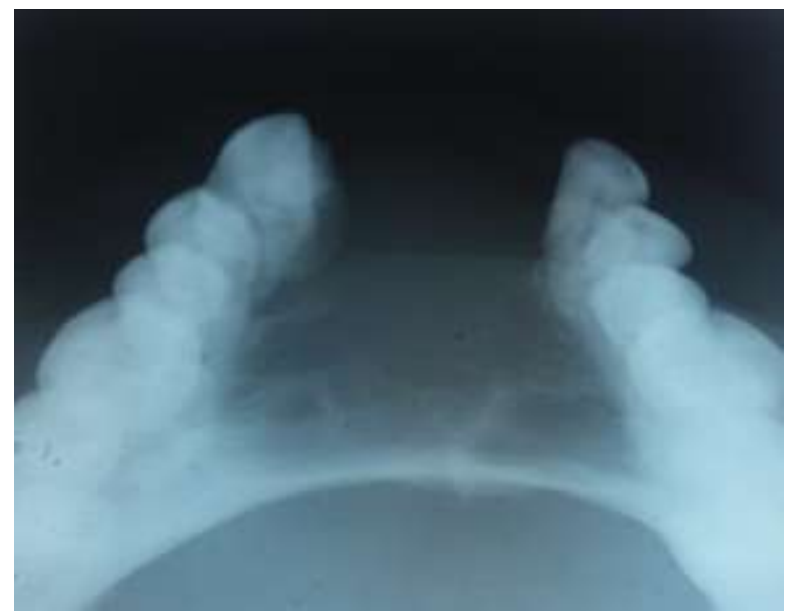

Fig 4.Mandibular anterior topographic occlusal radiograph showing angular internal loculations 


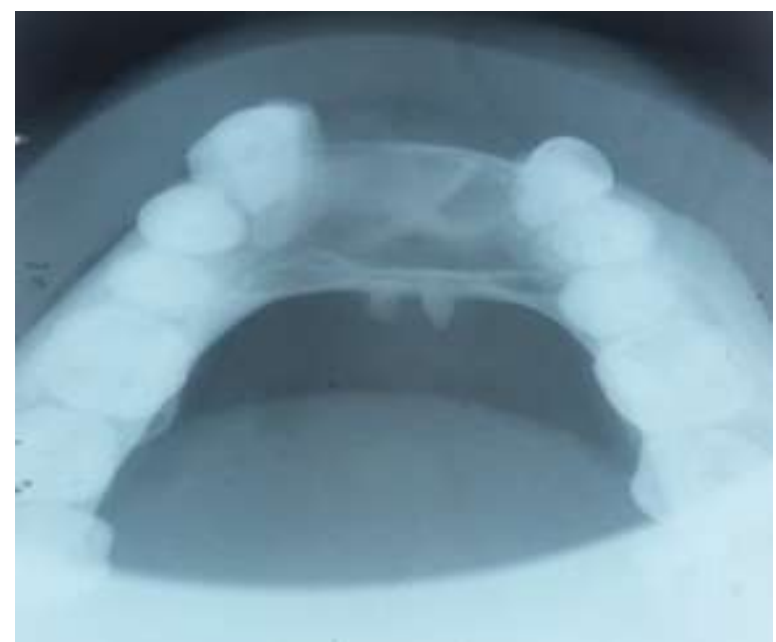

Fig 5.Anterior mandibular true occlusal view

FNAC, smear showed two clusters of basaloid cells with hyperchromatic nuclei and minimal cytoplasm, loose fibrillar stroma with minimal inflammatory cells. Some scattered squamous epithelial cells were also seen. Features were suggestive of a lesion of odontogenic origin.Incisional biopsy was performed which was followed by wide excision. Gross specimen showed soft tissue which was pearly white in colour with underlying brown areas. It was slimy in nature and had soft jelly like consistency. Microscopy revealed serial sections of $\mathrm{H} \& \mathrm{E}$ stained tissue showing spindle and stellate shaped cells present in a mucoid stroma. The spindle shaped cells were widely dispersed with elongated and delicate cytoplasmic cell processes. The collagen fibrils were very delicate. Stellate shaped cells were seen traversing between the muscle fibres. Perivascular hyalinization, minimal inflammatory cells and occasional mast cells were also seen. Submucosa showed skeletal muscle fibres and adipocytes. Microscopic features were suggestive of Odontogenic myxoma. (Fig 6 and Fig 7)
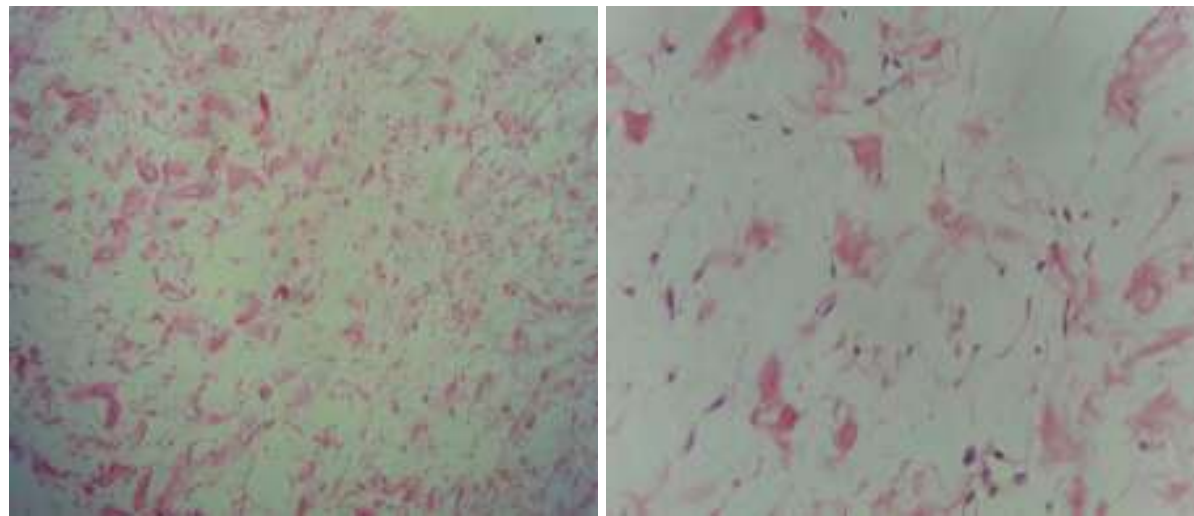

Fig 6. (left) and Fig 7 (right). $\mathrm{H}$ and $\mathrm{E}$ stained section showing stellate, spindle-shaped cells in an abundant, loose myxoid stroma

Based upon the clinical, radiographic and histopathologic findings, a final diagnosis of odontogenic myxoma of anterior mandible crossing the midline was made.

\section{Discussion}

Odontogenic myxoma is considered as a rare, locally invasive, benign tumour derived from embryonic mesenchymal structures of dental primordium including dental papilla, dental follicle or Hertwig's epithelial root sheath. Its sole occurrence in the tooth bearing areas of the jaws, frequent association with a missing or unerupted tooth, histologic similarity to dental papilla and occasional appearance of odontogenic epithelial islands backs its odontogenic origin.[6,7] WHO in 2013 grouped it under benign neoplasms arising from odontogenic ectomesenchyme with or without odontogenic epithelium.[8] Rudolf Virchow, in 1863 was probably the pioneer to describe histologic features of myxofibroma. However he didn't mention the lesions of the jaws particularly. Later, in 1947 Thoma and Goldman described odontogenic myxomas of the jaws.[6,9]It is otherwise called as myxofibroma or myxoma.[8]

Odontogenic myxoma accounts for around 3-6\% of all odontogenic tumours with a slightly higher frequency noted in America, Europe and Asia[3,10] It is more common in females of second and fourth 
decades of life. It is rare below 10 years of age and above 50 years, even though very few cases in an age range of 1to 73 years also have been reported in literature.[11] It is more common in posterior mandibular ramus region. $[3,10]$ However possibility of occurrence in maxilla, mandibular incisive area and condylar region should not be neglected.[10] Odontogenic myxoma rarely crosses the midline.[11] Present case shows lesion in anterior mandibular region crossing the midline in a 61 year old male patient. It is an asymptomatic, slowly growing lesion which is nonencapsulated and shows an infiltrative growth pattern which may lead to destruction of medullary bone, expansion of cortical bone and in late stages it may perforate and invade adjacent cortical bone and soft tissues.[12] It rarely cause tooth displacement and mobility of involved teeth. The biologic nature of the tumour hold responsible for its invasiveness.[13] Pain and pareasthesia are infrequent and thus patients may not perceive the presence of the lesion until it reaches a noticeable size. Progressive lesions may eventually cause neurologic disturbances.[3]

The typical radiographic appearance of a myxoma is a multilocular radiolucency with sharp, thin trabeculations which intersect at right angles giving rise to a "tennis racket" appearance. However, radiographic appearance varies from a unilocular appearance, which is more common in anterior jaw and in children to a multilocular "tennis racket", "soap bubble" or "honey comb" appearance.[14] It may also give rise to a "sun burst" or "hair brush" appearance when peripheral margins of the septa are arranged at right angles to the margins.[4,9] The mixed radiographic appearance of odontogenic myxoma may be attributed to residual bone and not due to new bone formation.[15] It may have well-defined non corticated, well-defined corticated, poorly defined or diffuse margins. [2] It may cause cortical expansion of jaw bones and tooth displacement rather than root resorption. Maxillary lesions are more aggressive and may invade maxillary antrum.[11] The tumour is often scalloped between the roots in tooth bearing areas.[10] Radiographic differential diagnoses considered are ameloblastoma, intraosseous hemangioma, central giant cell granuloma and odontogenic keratocyst. In the present case it appeared as a multilocular radiolucency with angular internal loculations resembling a "tennis racket" pattern.

Gross surgical specimen usually appears as a yellowish grey glistening or gelatinous nonencapsulated mass. Histopathologically the lesion contains spindle shaped, round or angular cells in an abundant loose myxoid stroma with minimum vascularity and few fine fibrillar material. Cells show finely granular, slightly basophilic cytoplasm with a well defined nucleus. Polymorphic cells, polymorphic or hyperchromatic nuclei and mitotic figures are rarely seen. Occasionally inflammatory infiltrate and remnants of odontogenic epithelium may also be seen. Odontogenic epithelium may rarely be surrounded by a narrow zone of hyalinization. Myxoid nerve sheath tumour, hyperplastic dental follicle and chondromyxoid fibroma can be considered as differential diagnosis after histopathologic examination.[4,9] Present case also showed a similar appearance. As the tumour is not radiosensitive, surgery is the treatment of choice. There is a lack of proper consensus regarding the type of surgery to be performed. Even though it has several advantages, considering the aggressive nature of tumour, its tendency to infiltrate into adjacent medullary and cortical bone, lack of capsule and high recurrence rate, radical treatment or block resection is advised over conservative treatment. An alternative conservative method by excision and curettage with liquid nitrogen cryotherapy has also been proposed.[3,11,16] As the lesion is notorious for recurrence, patient has to be periodically followed up.

\section{Conclusion}

To conclude, odontogenic myxoma is a rare, benign, locally invasive, asymptomatic odontogenic neoplasm routinely encountered in mandibular ramus region of females in second to fourth decades of life. Most oftenly it is detected only after it attains a considerable size. Radiographically, typical appearance is that of a multilocular radiolucency with fine, angular trabeculations. Histopathologically it consists of rounded and angular cells in an abundant mucoid stroma. Management by radical surgery and long term follow-up is required owing to its aggressive nature and tendency to recur. This case throws light on the necessity of considering odontogenic myxoma as a diagnostic possibility of a multilocular radiolucency even when it is an atypical location and age group.

\section{References}

[1]. Kawase-koga Y, Saijo H, Hoshi K, Takato T, Mori Y. Surgical management of odontogenic myxoma : a case report and review of the literature. BioMed Cent Res Notes. 2014;7:1-7.

[2]. Altug HA, Gulses A, Sencimen M. Clinico-Radiographic Examination of Odontogenic Myxoma with Displacement of Unerupted Upper Third Molar : Review of the Literature. Int J Morphol. 2011;29(5):930-3.

[3]. Manne RK, Kumar V, Sarath PV, Anumula L, Mundlapudi S, Tanikonda R. Case Report Odontogenic Myxoma of the Mandible. Case Rep Dent. 2012;2012:1-4.

[4]. Vasudevan V, Das UM, Manjunath V, Bavle RM, Sudhakar M, Kumar N, et al. Odontogenic Myxoma of the Maxilla : A Report of Unusual Pediatric Case. Int J Clincal Pediatr Dent. 2011;4(3):264-8.

[5]. Munjal M, Bhardwaj V, Garg B, Sood N. Odontogenic Myxoma Of The Maxilla: A Clinical Case Report And Review Of Literature. Otolaryngeology online J. 2013;3(2).

[6]. Ashoka CA. Odontogenic Myxoma Of Mandible: Report Of A Rare Case With Review Of Literature. IOSR J Dent Med Sci. 2016;15(1):35-40. 
[7]. Jindwani K, Nevaskar V, Agrawal D. Odontogenic Myxoma of Maxilla : Management and Follow-up of A Rare Case. Indian J Clin Pract. 2013;24(2):130-7.

[8]. Heichart P, Philipson H. Odontogenic tumours and allied lesions. In London: Quintessence Publishing Co.Ltd; 2004. p. 189-97.

[9]. Rani V, Kadar MKM, Babu A, Sankari L, Krishnasamy G. Odontogenic myxoma diagnostic dilemma : A case report and review of literature. J Cranio-Maxillary Dis. 2014;3(2):163-7.

[10]. Reddy SP, Naag A, Kashyap B. Odontogenic myxoma : Report of two cases. Natl J Maxillofac Surg. 2010;1(2):183-6.

[11]. Li T, Sun L, Luo H. Odontogenic Myxoma A Clinicopathologic Study of 25 Cases. Arch Pathol Lab Med. 2006;130(December):1799-806.

[12]. Tuji M, Eid NLM, Aquilino RN, Molina OF, Joo HY, Neto FH. Odontogenic myxoma in the maxilla: A case report and characteristics on CT and MR. Oral Oncol Extra. 2006;42:133-6.

[13]. Hemavathy S, Kumar DV. Odontogenic Myxoma . A case report. IOSR J Dent Med Sci. 2014;13(3):46-9.

[14]. Gupta S, Grover N, Kadam A, Gupta S, Sah K, Sunitha JD. Odontogenic myxoma. Natl J Maxillofac Surg. 2013;4(1):81-3.

[15]. Kaffe I, Naor H, Büchner A. Clinical and radiological features of odontogenic myxoma of the jaws. Dentomaxillofacial Radiol. 1997;26(January):299-303.

[16]. Ayranc F, Ömezli MM, Rastgeldi OZ, Duman A. Odontogenic Myxoma Located in The Mandible: A Case Report. Middle Black Sea J Heal Sci. 2015;1(December):25-8. 